\title{
Acetylcholine from Visual Circuits Modulates the Activity of Arousal Neurons in Drosophila
}

\author{
Nara I. Muraro and M. Fernanda Ceriani \\ Fundación Instituto Leloir, Instituto de Investigaciones Bioquímicas, Buenos Aires-Consejo Nacional de Investigaciones Científicas y Técnicas, C1405BWE \\ Buenos Aires, Argentina
}

Drosophila melanogaster's large lateral ventral neurons (lLNvs) are part of both the circadian and sleep-arousal neuronal circuits. In the past, electrophysiological analysis revealed that lLNvs fire action potentials (APs) in bursting or tonic modes and that the proportion of neurons firing in those specific patterns varies circadianly. Here, we provide evidence that lLNvs fire in bursts both during the day and at night and that the frequency of bursting is what is modulated in a circadian fashion. Moreover, we show that lLNvs AP firing is not only under cell autonomous control, but is also modulated by the network, and in the process we develop a novel preparation to assess this. We demonstrate that lLNv bursting mode relies on a cholinergic input because application of nicotinic acetylcholine receptor antagonists impairs this firing pattern. Finally, we found that bursting of lLNvs depends on an input from visual circuits that includes the cholinergic L2 monopolar neurons from the lamina. Our work sheds light on the physiological properties of lLNvs and on a neuronal circuit that may provide visual information to these important arousal neurons.

Key words: acetylcholine; arousal; bursting neuron; Drosophila; electrophysiology; ILNv

\section{Significance Statement}

Circadian rhythms are important for organisms to be able to anticipate daily changes in environmental conditions to adjust physiology and behavior accordingly. These rhythms depend on an endogenous mechanism that operates in dedicated neurons. In the fruit fly, the large lateral ventral neurons (lLNvs) are part of both the circadian and sleep-arousal neuronal circuits. Here, we provide new details about the firing properties of these neurons and demonstrate that they depend, not only on cell-autonomous mechanisms, but also on a specific neurotransmitter derived from visual circuits. Our work sheds light on the physiological properties of ILNvs and on a neuronal circuit that may provide visual information to these important arousal neurons.

\section{Introduction}

Circadian rhythms are important for organisms to be able to anticipate daily changes in environmental conditions to adjust physiology and behavior accordingly. In animals, circadian rhythms are determined by the interaction of a network of clock neurons that express a set of molecular components that establish transcriptional-translational feedback loops that ensure near

\footnotetext{
Received April 22, 2015; revised 0ct. 9, 2015; accepted 0ct. 15, 2015.

Author contributions: N.I.M. and M.F.C. designed research; N.I.M. performed research; N.I.M. analyzed data; N.I.M. and M.F.C. wrote the paper.

This work was supported by Agencia Nacional de Promoción Científica y Tecnológica (Grant PICT-2011-2185 to M.F.C. and Grant PICT-2011-2364 to N.I.M.). M.F.C. and N.I.M. are members of the Argentine Research Council for Science and Technology (CONICET). We thank Sofia Polcowñuk for help with statistical analysis, Esteban Beckwith for help with sleep quantification software, and Lia Frenkel for fruitful discussion and ideas.

The authors declare no competing financial interests.

Correspondence should be addressed to either of the following: Nara I. Muraro, Instituto de Investigación en Biomedicina de Buenos Aires (IBioBA), Partner of the Max Planck Society, CONICET, Godoy Cruz 2390, C1425FQD Buenos Aires, Argentina, E-mail: nmuraro@leloir.org.ar; or M. Fernanda Ceriani, Fundación Instituto Leloir, IIBBACONICET, Av. Patricias Argentinas 435, C1405BWE Buenos Aires, Argentina, E-mail: fceriani@leloir.org.ar.

DOI:10.1523/JNEUROSCI.1571-15.2015

Copyright $\odot 2015$ the authors $\quad 0270-6474 / 15 / 3516315-13 \$ 15.00 / 0$
}

24 h cycling (Hardin, 2011; Ozkaya and Rosato, 2012). In Drosophila, these processes are highly active in $\sim 150$ clock neurons, grouped in clusters according to their anatomical position, comprising three dorsal neuron clusters termed DN1 to DN3 and four lateral groups divided in small (sLNvs) and large (lLNvs) ventral lateral, dorsal lateral, and lateral posterior neurons.

Current views in circadian rhythms biology highlight the importance of the clock neuronal network as a whole for circadian timing and for the plasticity that the underlying interaction ensures (Welsh et al., 2010; Muraro et al., 2013). To gain insight into the physiology of clock neurons in Drosophila, a preparation suitable for whole-cell patch-clamp electrophysiology ex vivo has been exploited (Park and Griffith, 2006; Cao and Nitabach, 2008; Sheeba et al., 2008a; Depetris-Chauvin et al., 2011; Fogle et al., 2011; McCarthy et al., 2011; Li et al., 2013; Liu et al., 2014; Seluzicki et al., 2014; Tabuchi et al., 2015). However, little is known about the physiological features of Drosophila clock neurons.

An interesting property of the lLNvs is that they are able to fire action potentials (APs) in a bursting mode, in a tonic mode, or do not fire APs spontaneously and the proportion varies in a circadian 
manner, with the high-activity bursting mode being more prevalent during the day and the tonic and silent modes more prevalent at night (Sheeba et al., 2008a). The fact that ILNvs can fire APs in a bursting mode is consistent with their being peptidergic neurons because neuropeptide release may be evoked by this type of highfrequency firing (Dutton and Dyball, 1979; Liu et al., 2011). LNvs communicate through a neuropeptide termed pigment dispersing factor (PDF), which plays roles as a circadian synchronizing signal when released from sLNvs (Lin et al., 2004) and as an arousal signal when released from ILNvs (Sheeba et al., 2008b; Chung et al., 2009). In the case of sLNvs, daily cycling of PDF immunoreactivity has been demonstrated in their axon terminals projecting to the dorsal protocerebrum (Park et al., 2000). Bursting of sLNvs has not been reported yet, but we have performed recordings of this neuronal group and observed this firing mode (N.I.M. and M.F.C., unpublished data). Further research is necessary to determine whether bursting frequency varies circadianly in sLNvs; however, by means of genetically encoded voltage sensors, it has been shown that depolarization events with durations compatible with neuronal bursting are more frequent in the morning than in the evening in sLNvs (Cao et al., 2013).

Circadian variation of neuronal firing frequency as an output of the Drosophila molecular clock, as has been shown in mammals (Colwell, 2011), is indeed an attractive possibility, prompting us to explore the underlying molecular mechanisms. In the process, we found that the $1 \mathrm{LNv}$ firing mode reflects, not the variation of gene expression controlled by the clock, but rather it largely depends on input from the neuronal network. In fact, when recordings are established rapidly after brain dissection, ILNvs always fire APs organized in bursts regardless of the time of day. We propose that, in the intact organism, it is the frequency of bursting that determines the degree of peptide released at different times of the daily cycle. In addition, we found that ILNv bursting depends on a cholinergic input from circuits involved in visual processing including the L2 monopolar neurons. Overall, our work sheds light on the nature of lLNv firing and on a previously undescribed neuronal circuit from L2 to lLNvs that may provide visual information for arousal control.

\section{Materials and Methods}

Strains and fly rearing. pdf-GAL4 (II chromosome), UAS-CD8GFP (III chromosome), Gmr-GAL4 (II chromosome), and UAS-TrpA1 (II chromosome) were obtained from the Bloomington Stock Center. $p d f$-DsRed (III chromosome) was provided by J. Blau (New York University). L2GAL4 (III chromosome) was provided by O. Shafer (University of Michigan). L1-GAL4 (II chromosome) was provided by Jens Rister (New York University). Lai split GAL4 (R92A10AD attP40; R17D06DBD attp2, II and III chromosomes) was provided by Aljoscha Nern (Janelia Research Campus-HHMI). LexAop-CD4spGFP ${ }^{11}$ (II chromosome) and UASCD4spGFP ${ }^{1-10}$ (III chromosome) were provided by K. Scott (University of California-Berkeley). pdf-LexA (II chromosome) was provided by M. Rosbash (Brandeis University). A recombinant $p d f$-LexA,LexAop$C D 4 s p G F P^{11}$ (II chromosome) previously generated in the laboratory (Gorostiza et al., 2014) was used for GFP Reconstitution Across Synaptic Partners (GRASP) analysis. Flies were grown and maintained at $25^{\circ} \mathrm{C}$ in standard cornmeal medium under 12:12 h light:dark cycles. For experiments involving fly crosses containing TrpA1, flies were raised at $22^{\circ} \mathrm{C}$.

Electrophysiology. Three- to 10-d-old female flies were anesthetized with a brief incubation of the vial on ice and brain dissection was performed on external recording solution consisting of the following (in $\mathrm{mm}$ ): $101 \mathrm{NaCl}, 3 \mathrm{KCl}, 1 \mathrm{CaCl}_{2}, 4 \mathrm{MgCl}_{2}, 1.25 \mathrm{NaH}_{2} \mathrm{PO}_{4}, 5$ glucose, and $20.7 \mathrm{NaHCO}_{3}, \mathrm{pH} 7.2$, with an osmolarity of $250 \mathrm{mmol} / \mathrm{kg}$ (based on solution used by Cao and Nitabach, 2008). After removal of the proboscis, air sacks, and head cuticle, the brain was routinely glued ventral side up to a sylgard-coated coverslip using a few $\mu$ ls of tissue adhesive $3 \mathrm{M}$
Vetbond. The time from anesthesia to the establishment of the first successful recording was $\sim 15-19$ min spent as following: 5-6 min for the dissection, 4-5 min for the protease treatment to remove the brain's superficial glia, and 6-8 min to fill and load the recording electrode onto the pipette holder, approach the cell, achieve the gigaohm seal, and open the cell into whole-cell configuration to start recording. For the novel dissection preserving the eyes shown in Figure 5, the head cuticle was carefully detached from the eye rim using sharp forceps resulting in the conservation of the whole structure as shown in Figure 5A. This dissection took, on average, 3.3 min longer to perform than the original one (original dissection duration: $5.5 \pm 0.2 \min n=10$, novel dissection duration: $8.8 \pm 1.3 \min n=20, t$ test $p<0.01$ ). LNvs were visualized by red fluorescence in $p d f$-DsRed or green fluorescence in $p d f$-GAL4; UASCD8GFP (referred to in the text as $p d f>$ CD8GFP) using a Leica DM LFS upright microscope with $63 \times$ water-immersion lens and TK-LED illumination system (TOLKET). Once the fluorescent cells were identified, cells were visualized under infrared differential interference contrast using a Hamamatsu ORCA-ER camera and Micro Manager version 1.4.14 software. ILNvs were distinguished from sLNvs by their size and anatomical position. To allow the access of the recording electrode, the superficial glia directly adjacent to lLNvs were locally digested with protease XIV solution $(10 \mathrm{mg} / \mathrm{ml}$; P5147; Sigma-Aldrich) dissolved in external recording solution. This was achieved using a glass capillary (pulled from glass of the type GC100TF-10; Harvard Apparatus) and gentle massage of the superficial glia with mouth suction to render the underling cell bodies accessible for the recording electrode with minimum disruption of the neuronal circuits. Whole-cell recordings were performed using thickwalled borosilicate glass pipettes (GC100F-10; Harvard Apparatus) pulled to 6-7 M $\Omega$ using a horizontal puller (P-1000; Sutter Instruments) and fire polished to 9-12 M $\Omega$. Recordings were made using an Axopatch 200B amplifier controlled by pClamp version 9.0 software via a Digidata 1322A analog-to-digital converter (Molecular Devices). Recording pipettes were filled with internal solution containing the following (in $\mathrm{mm}$ ): 102 potassium gluconate, $17 \mathrm{NaCl}, 0.085 \mathrm{CaCl}_{2}, 0.94$ EGTA and 8.5 HEPES, pH 7.2 with an osmolarity of $235 \mathrm{mmol} / \mathrm{kg}$ (based on the solution used by Cao and Nitabach, 2008). Gigaohm seals were accomplished using minimal suction followed by break-in into whole-cell configuration using gentle suction in voltage-clamp mode with a holding voltage of $-80 \mathrm{mV}$. Gain of the amplifier was set to 1 during recordings and a 5 $\mathrm{kHz}$ low-pass Bessel filter was applied throughout. Spontaneous firing was recorded in current-clamp $(I=0)$ mode. Analysis of traces was performed using Clampfit version 10.4 software. Bursting frequency was calculated as the number of bursts in the first minute of recording. For AP firing rate calculation, the event detection tool of Clampfit version 10.4 was used. In many cases, we were able to see the two different AP sizes reported previously (Cao and Nitabach, 2008); however, for AP firing rate calculation, only the large APs were taken into account. Traces shown in figures were filtered offline using a low-pass boxcar filter with smoothing points set to 9. Perfusion of external saline in the recording chamber was achieved using a peristaltic pump (MasterFlex C/L). Curare (200 $\mu \mathrm{M}$ in external saline; T-2379; Sigma-Aldrich) or mecamylamine (10 $\mu \mathrm{M}$ in external saline; 9020; Sigma-Aldrich) were bath perfused. For the TrpA1 experiments, after a few minutes of recording in currentclamp mode to record the basal bursting frequency, $10 \mathrm{ml}$ of warmed external solution was perfused, resulting in the increase of bath temperature presented in Figure $6 G$ (bottom). Perfusion of solution at room temperature was continued when warm solution had finished. For Figure $6 G$, bursting frequency was calculated every $30 \mathrm{~s}$.

Immunohistochemistry and GRASP analysis. Dissection and immunostaining of adult fly brains were performed as described previously (Depetris-Chauvin et al., 2011). To detect GFP reconstitution, a mouse monoclonal anti-GFP antibody from Sigma-Aldrich (G6539) that recognized the reconstituted GFP molecule, but not the GFP ${ }^{1-10}$ or GFP ${ }^{11}$ fragments alone, was used at a 1:10 dilution as described previously (Gorostiza et al., 2014). To focus the search of GRASP near lLNVs projections in the optic lobes, a rabbit anti-PDF (custom made by NeoMPS) was used at 1:500 dilution. To evidence the expression patterns of the GAL4 drivers used for GRASP, they were crossed to UAS-CD8GFP and the progeny stained with anti-GFP primary antibody (GFP-1020; Aves Labs) together with the anti- 
A

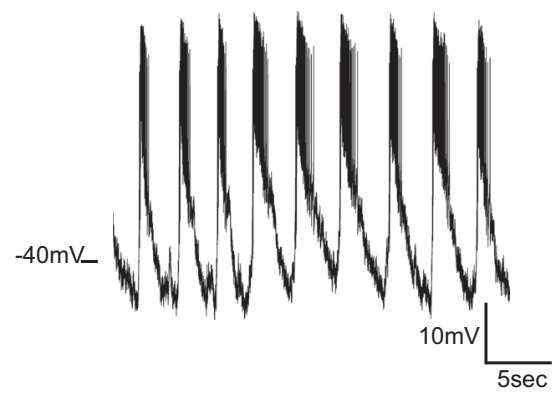

D

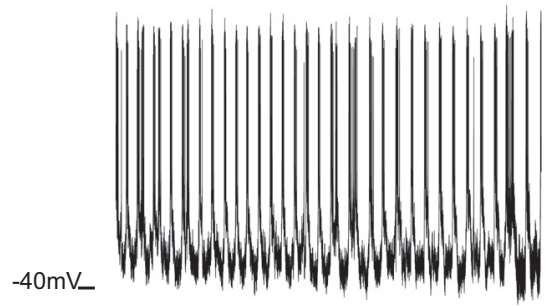

$10 \mathrm{mv} \frac{}{10 \mathrm{sec}}$

$\min 0-1$

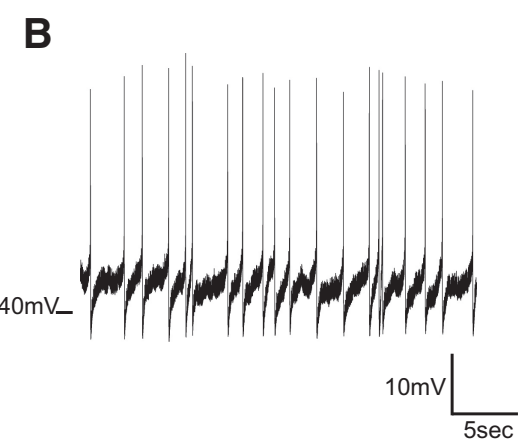

E
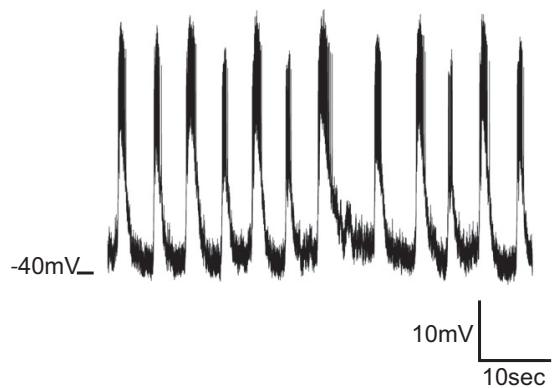

$\min 15-16$

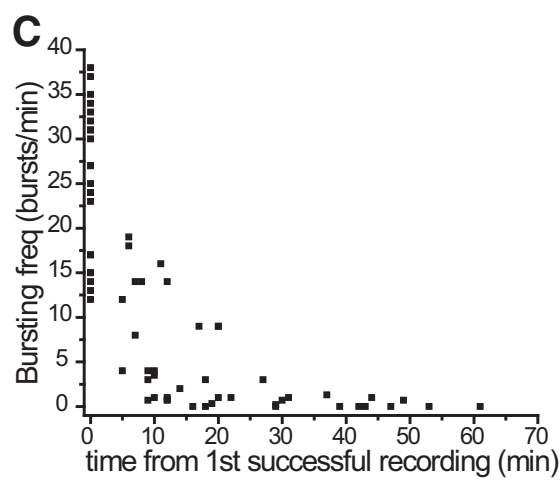

$\mathbf{F}$

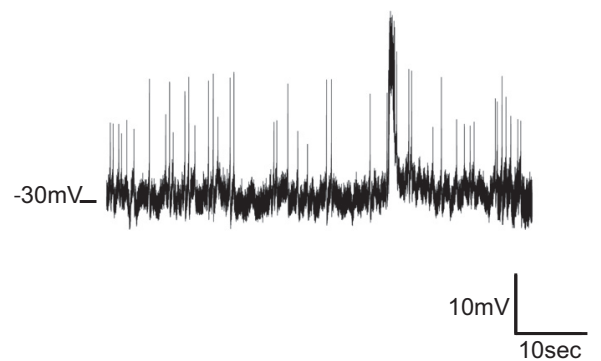

$\min 30-31$

Figure 1. ILNvs are bursting neurons. Whole-cell patch-clamp recordings on control ILNvs ( $p d f$-DsRed). $\boldsymbol{A}$, Representative recording of an ILNv firing APs in bursts. $\boldsymbol{B}$, Representative recording of a ILNv presenting tonic firing of APs. C, ILNv bursting frequency correlates with the time elapsed since dissection. All ILNvs recorded were spontaneously firing APs. Zero burst frequency represents a tonic firing neuron. Time 0 is the minimum time from dissection to the establishment of the first successful recording ( $\sim 15-19$ min from anesthesia). Each square corresponds to the bursting frequency at the time of the recording of individual ILNvs; some were quickly recorded (time 0 ) and others were aged and only then were the recordings established. $n=67$. Zeitgeber time of recordings was distributed around the whole $24 \mathrm{~h}$ cycle. $\boldsymbol{D}-\boldsymbol{F}$, Fragments of a long recording of an individual ILNv that starts with a bursting frequency of 31 bursts/min (Fig. 1D), decays to 12 burst/min after $15 \mathrm{~min}$ (Fig. 1E), and shows only 1 burst/min after $30 \mathrm{~min}$ (Fig. $1 F$ ). Note that the quality of the recording degrades with prolonged times in whole-cell configuration, probably due to dialyzation of the cytoplasm. However, this does not preclude the visualization of the progressively more infrequent bursts. $\boldsymbol{D}-\boldsymbol{F}$ correspond to different parts of the same recording.

PDF antibody. Secondary antibodies used were Cy5-conjugated anti-rabbit, Cy2-conjugated anti-mouse, or Cy2-conjugated anti-chicken (Jackson ImmunoResearch) at 1:500 dilutions. Images were taken on Zeiss LSM 710 microscope. After acquisition, images were processed with Fiji software, an ImageJ-based image-processing environment (Schindelin et al., 2012).

Sleep analysis. Female flies were housed socially in vials from eclosion at $22^{\circ} \mathrm{C}$ under 12:12 h light:dark cycles until they were 4 to $7 \mathrm{~d}$ old and afterward transferred to $65 \times 5 \mathrm{~mm}$ glass tubes (Trikinetics) containing normal cornmeal. Tubes were loaded onto DAM monitors and locomotor activity was assessed using the DAM system for $2 \mathrm{~d}$ at $22^{\circ} \mathrm{C}, 1 \mathrm{~d}$ at $29^{\circ} \mathrm{C}$ for TrpAl activation, and then returned to $22^{\circ} \mathrm{C}$ for another day, always under $12: 12 \mathrm{~h}$ light:dark cycles. The DAM System binning time was set to $1 \mathrm{~min}$. Sleep was defined as no movement for 5 min (Hendricks et al., 2000; Shaw et al., 2000). pySolo software (Gilestro and Cirelli, 2009) was used to calculate sleep from locomotor activity data; to build graphs of sleep for $30 \mathrm{~min}$ as a function of the time of day; to get measurements of total sleep (minutes), day sleep (minutes), and night sleep (minutes); and to get an activity index (defined as the total activity counts divided by the total time spent awake in a $24 \mathrm{~h}$ period) of each individual fly. Behavioral experiments were conducted at least three times with 29-32 individuals per genotype.

Statistics. According to necessity, Student's $t$ test and one- or two-way ANOVA analyses were performed. Student's $t$ test and one-way ANOVA were performed using OriginPro8 software. Two-way ANOVA were performed using Rstudio 3.0.1 software.

\section{Results}

lLNvs are bursting neurons

Previous reports of recordings performed in whole-cell patchclamp mode in ex vivo Drosophila brains described that lLNvs can be found firing APs in bursting or tonic patterns (Cao and Nitabach, 2008; Sheeba et al., 2008a) or may be silent (Park and
Griffith, 2006; Cao and Nitabach, 2008; Sheeba et al., 2008a). Using the same recording technique, we indeed observed the two reported ILNv firing modes (Figs. $1 A, B$ ); however, we did not at any time of the day find silent cells. Recordings were performed on $p d f$-DsRed flies in which expression of the fluorescent protein DsRed is only directed to LNv (PDF-positive) cells. A thorough analysis of the firing behavior of a large number of recordings allowed us to observe that $\mathrm{lLNv}$ firing mode depended on the time from dissection to recording. Figure $1 C$ shows the frequency of bursts of lLNvs as a function of this time, with zero bursting frequency representing a tonic firing neuron. In Figure $1 C$, zero in the time axis $(x)$ indicates the time of the first successfully recorded cell in a freshly dissected brain. Each square corresponds to the bursting frequency calculated from the first minute in current-clamp mode of individual cells recorded at increasing times after dissection at zeitgeber times distributed around the entire $24 \mathrm{~h}$ cycle. Figure $1 C$ shows that no tonic firing $1 \mathrm{LNvs}$ are found at the onset of the experiment and that the frequency of bursts decays quickly with time (within minutes).

We reasoned that the decay in bursting frequency could depend on the age of the preparation or, alternatively, bursting of a given ILNv could depend on the integrity of the other ipsilateral ILNvs that is lost as more recordings are attempted or performed within the same preparation. To test our hypotheses, we performed several different experiments. First, we performed longer recordings (25-30 $\mathrm{min}$ ) of individual lLNvs to determine whether burst frequency decreased with time in an individual cell. Figure 1, $D-F$, shows that this was indeed the case because a 
ILNv beginning with a bursting frequency of 31 bursts/min (Fig. 1D) decayed to 12 after $15 \mathrm{~min}$ (Fig. $1 E$ ) and showed only 1 after $30 \mathrm{~min}$ (Fig. $1 F$ ). An independent experiment designed to test this possibility consisted of performing whole-cell recordings on deliberately aged preparations. Either the brain was dissected, treated with protease to remove superficial glia, and then kept in the perfusion chamber for 30-40 min before recordings of several lLNvs were performed or it was dissected, kept in the perfusion chamber for 30-40 min, and only then treated with protease right before recording. In both cases, ILNvs from aged preparations all displayed tonic firing or had a very low bursting frequency $(<2$ bursts/min, data not shown). No silent cells were recorded even in preparations older than $1 \mathrm{~h}$; cells were alive because they presented negative resting membrane potential and were actively firing APs.

To further test the possibility that the bursting mode required integrity within the $1 \mathrm{LNv}$ cluster, two to three lLNvs were removed by suction during the protease treatment and tested to determine whether the first cell recorded from the one to two remaining ipsilateral lLNvs were still bursting. This procedure did not prevent recording a bursting neuron at first. Additional control experiments confirmed that burst frequency decay is not genotype specific (because $p d f>$ CD8GFP flies followed the same trend; data not shown) and does not depend on technical aspects of the preparation (i.e., carbogen bubbling of the perfusion saline, which was not used on a regular basis) or the method used to immobilize the brain before recording (i.e., fine dissection pins). In all cases, we saw the decay in bursting frequency that correlated with the age of the preparation.

All of these data together suggest that lLNvs may not fire APs physiologically in a tonic mode, but rather this activity pattern may reflect a quick degradation of the preparation due to the manipulation and is perhaps a consequence of the gradual loss of a synaptic input that enables lLNvs to fire APs organized in bursts.

\section{lLNv firing mode depends on the time of day}

Our data suggest that it is the brain manipulation that causes the decrease in burst frequency; however, it has been reported that ILNvs are more active during the day (with a higher proportion of bursting neurons) than during the night (with a higher proportion of tonic and silent neurons) (Sheeba et al., 2008a; Li et al., 2013).

To reconcile our data with the published data, we analyzed the proportion of bursting and tonic lLNvs at different zeitgeber time ranges without taking into account the time elapsed since dissec-
B

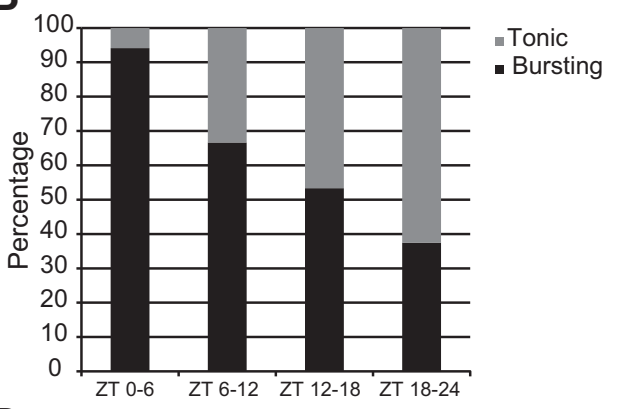

D

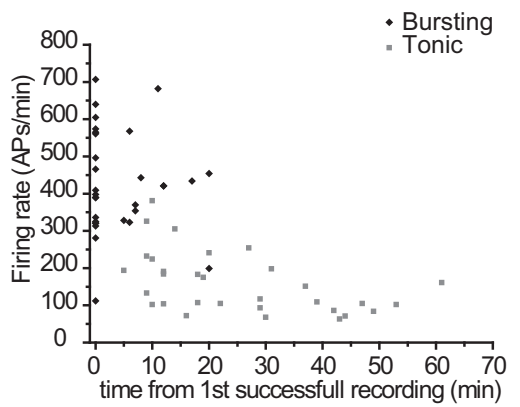

$\mathbf{F}$

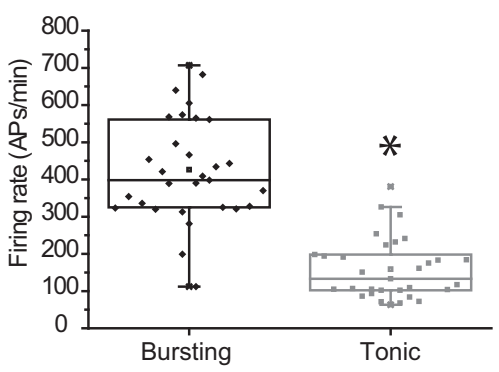

Figure 2. ILNv firing mode depends on the time of day. $\boldsymbol{A}$, Distribution of ILNv bursting frequency changes throughout the day regardless of the time taken from dissection to recording. During the day, higher frequency of bursting and a smaller proportion of roportion of tonic neurons was found. Only recordings performed within the initial 30 min were included in this analysis. $n=57$. , Proportion of bursting and tonic ILNvs found at different times of the day. Only recordings performed within the initial $30 \mathrm{~min}$

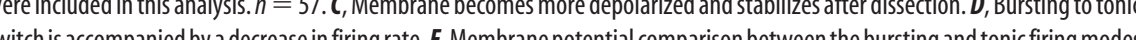
(same data as in $\boldsymbol{C}$ ). $\boldsymbol{F}$, Firing rate comparison between the bursting and tonic firing modes (same data as in $\boldsymbol{D}$ ). For $\boldsymbol{C}-\boldsymbol{F}, n_{\text {Bursting }}=$ $30, n_{\text {Tonic }}=31$. Asterisks indicate significant differences $(p<0.05)$ after a Student's $t$ test. Bursting neurons were defined as presenting $>5$ bursts/min and tonic neurons as having $<5$ bursts/min. Genotype: $p d f$-DsRed.

tion. We restricted the analysis to those recordings performed within the initial $30 \mathrm{~min}$ of the preparation because these were more likely to have been used by others in the past. Under these conditions, the proportion of firing patterns was similar to the reported ones, with a higher percentage of bursting lLNvs during the day (particularly during the morning) and more tonic lLNvs at night (Fig. 2A,B). For this analysis bursting neurons were defined as presenting $>5$ bursts/min and tonic neurons as having $<5$ bursts/min. The need for this categorization arose from the fact that very-low-frequency bursting neurons fired tonically during the interburst periods and therefore were effectively tonic firing neurons most of the time. In contrast to a previous report (Sheeba et al., 2008a), we found differences in membrane potential between the bursting and tonic firing modes (Fig. 2C,E), although this was correlated with a higher firing rate in bursting neurons than in tonic ones (Fig. $2 D, F$ ), as reported previously (Sheeba et al., 2008a). 
As mentioned before, despite the fact that tonic firing does not appear to be a physiological state of lLNvs in vivo, the observation that a larger proportion of tonically firing neurons can be recorded at night may reflect a basal lower bursting frequency modulated by a differential input from the neuronal network.

\section{Cholinergic input is lost in the ex vivo preparations}

It has been reported previously that LNvs can respond to acetylcholine (ACh). In the larval circadian system, light cues detected by the Bolwig's organ are communicated to the larval LNvs through ACh (Wegener et al., 2004). Moreover, using genetically encoded sensors in the adult, it has been shown that LNvs respond to cholinergic agonists with increases in intracellular $\mathrm{Ca}^{2+}$ and cAMP (Lelito and Shafer, 2012). In all cases, these responses have been reported to be mediated by nicotinic ACh receptors.

Electrophysiological recordings of lLNvs in the presence of cholinergic agonists show that, as expected for an excitatory neurotransmitter, they respond with depolarization of their membrane (McCarthy et al., 2011). In those early reports, it was concluded that cholinergic input was direct onto the lLNvs and that the resulting current had a reversal potential compatible with that of nicotinic receptors; in addition, bath application of the nicotinic antagonist curare $(200 \mu \mathrm{M})$ eliminated AP firing almost immediately and did not produce a change of membrane potential (McCarthy et al., 2011). However, we found that bath application of curare on brains dissected during the daytime did not eliminate AP firing; instead, it produced a change in the firing pattern of control ( $p d f$-DsRed) lLNvs from bursting to tonic (Fig. $3 A, B$ ), drastically reducing the firing rate of the neuron (Fig. $3 E$ ) but not eliminating it. Moreover, curare application resulted in a significant depolarizing change in membrane potential that accompanied the bursting to tonic switch in AP firing. To quantify the change in membrane potential, we compared the trough of the membrane potential between bursts in the bursting mode, with the resting membrane potential in the tonic mode (Fig. $3 D$ ). Interestingly, oscillations in membrane potential onto which bursts are mounted disappeared upon treatment with curare (Fig. 3B), indicating that bursting of lLNvs depended on a cholinergic input. We found the treatment with curare difficult to wash, with both membrane potential and AP firing not returning to basal levels. However, in some cases, we did see a partial reemergence of bursting behavior (Fig. 3C). To explore this phenomenon further, we tested the effect of curare on brains of flies of a different control genotype, $p d f>\operatorname{CD} 8 \mathrm{GFP}$ (Fig. $3 F, G$ ), and the influence of an alternative nicotinic ACh receptor antagonist, mecamylamine $(10 \mu \mathrm{M})$ (Fig. $3 H, I)$. In both cases, we observed a switch from bursting to tonic along with depolarization of the membrane. The degree of membrane depolarization and AP firing rate reduction vary in the two genotypes tested, however, the bursting to tonic firing switch occurred consistently in all the ILNvs tested upon perfusion of either nicotinic ACh receptor antagonist.

We then assessed the effect of curare application on ILNvs from brains dissected at zeitgeber times between 14 and 20 (nighttime). Because recordings were established quickly, all ILNvs were firing APs in bursting mode, albeit with a lower bursting frequency and lower AP firing rate, as expected. lLNvs responded to curare application in a similar manner during the day and at nighttime, with a switch from bursting to tonic firing (Fig. 4A, B), depolarization of the membrane (Fig. $4 C$ ), and a decrease in AP firing rate (Fig. 4D). However, at nighttime, a subset (three of eight recorded cells) of the lLNvs stopped firing after becoming tonic (Fig. $4 F-H$ ). This finding is consistent with the effect of cholinergic inhibition depending on the initial activity level of the neuron and partly reconciles our results with those reported previously by McCarthy et al. (2011).

Our results are consistent with a role of ACh in maintaining bursting of lLNvs. However, bath application of the nicotinic ACh receptor antagonists precludes any claim that ACh is acting on them directly. The fact that cholinergic antagonist application generates a bursting to tonic switch of AP firing in lLNvs, together with our previous data indicating that a similar switch from bursting to tonic is also produced (albeit at a slower pace) as the preparation is maintained ex vivo, suggests that a cholinergic input upstream of the lLNvs supports their bursting behavior.

\section{Synaptic input from the eye enables $1 \mathrm{LNv}$ bursting behavior}

Given lLNvs recognized role as arousal neurons (Shang et al., 2008; Sheeba et al., 2008b) and the localization of their widespread projections throughout the optic lobes, we hypothesized that the cholinergic input lost in the ex vivo preparation could derive from the eye, a structure that was removed in the standard preparation in which the electrophysiological recordings were performed. To test this hypothesis, we developed a new brain preparation in which the eyes were preserved (Fig. 5A). Given the fragility of the connections and the difficulty inherent to this dissection, it should be kept in mind that is it possible that some of the connections between the retina and the optic neuropiles may still be compromised under these conditions. Figure $5 B$ shows lLNv bursting frequency decay comparing the old and new preparations. It can be observed that preserving connectivity to the eyes protects to some extent the preparation from deterioration; bursting frequency still declines, but decay is slowed down significantly. Figure $5 C$ displays data from Figure $5 B$ as a box chart to allow better appreciation of the similitudes and differences of bursting frequency decay in the two preparations. It is worth pointing out that there are no statistically significant differences in the initial bursting frequency of either preparation; the differences lie in the progression of bursting decay with time. However, because the generation of the preparation that preserves the connection to the eyes is to some extent more cumbersome, a slight decay in bursting frequency might already have taken place. This result strongly suggests that synaptic inputs from the eye promote bursting of the lLNvs.

\section{L2 lamina neurons are part of the circuit that promotes lLNv bursting}

Because lLNvs play a role as arousal neurons, it would not be surprising if they received inputs from visual processing centers. Therefore, we hypothesized that visual information already processed by interneurons of the optic neuropiles could be the source of the signal that promotes bursting of the lLNvs. This would also account for the timing of the decay of ILNv bursting; if it depended on a direct connection from photoreceptors to ILNvs, bursting lLNvs would not have been detected in preparations lacking the eyes. Moreover, we have determined that bursting requires a cholinergic input, but photoreceptors in Drosophila use histamine as a neurotransmitter (Pollack and Hofbauer, 1991). Therefore, the source of ACh that promotes ILNv bursting may lie within the neuronal circuits of the optic neuropiles.

L2 lamina neurons receive direct input from outer photoreceptors (R1-R6) and project their axon toward the medulla. They constitute a first step in visual processing for motion detection and are cholinergic (Rister et al., 2007; Kolodziejczyk et al., 2008; Takemura et al., 2008). We hypothesized that L2 neurons could be the source of the ACh that promotes bursting of lLNVs. To test 
A

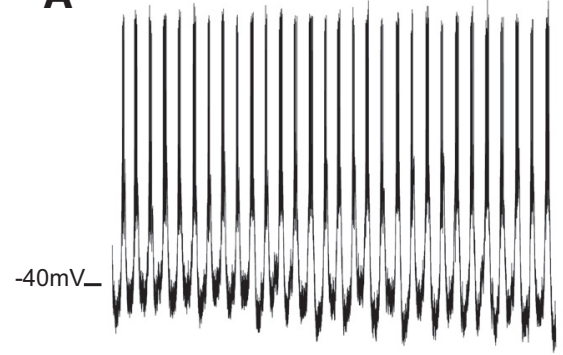

$10 \mathrm{mV} \frac{}{10 \mathrm{sec}}$

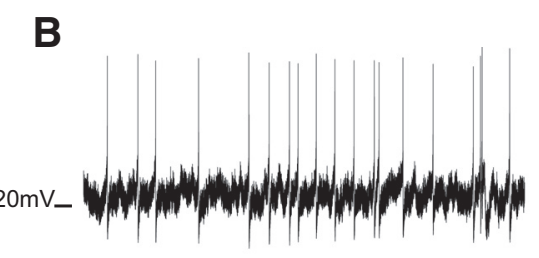

curare

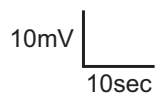

\section{C}

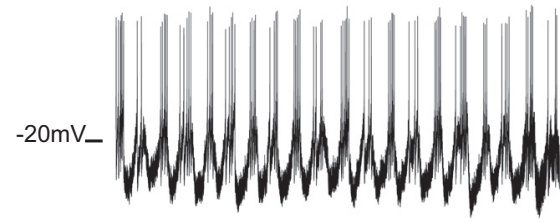

wash

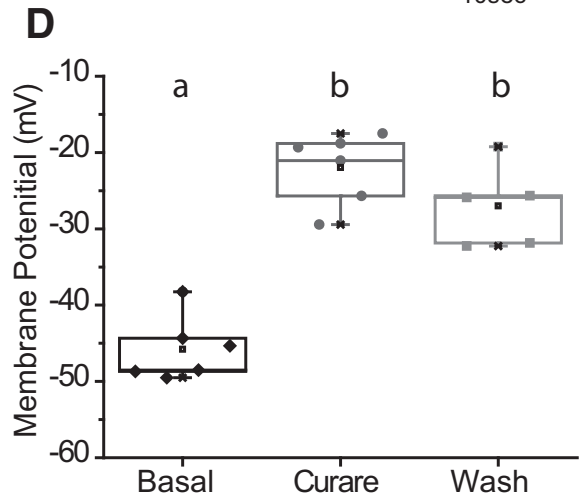

E

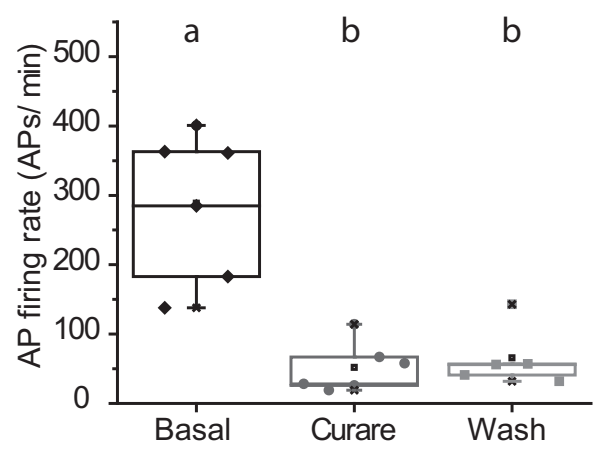

$10 \mathrm{mV} \frac{}{10 \mathrm{sec}}$

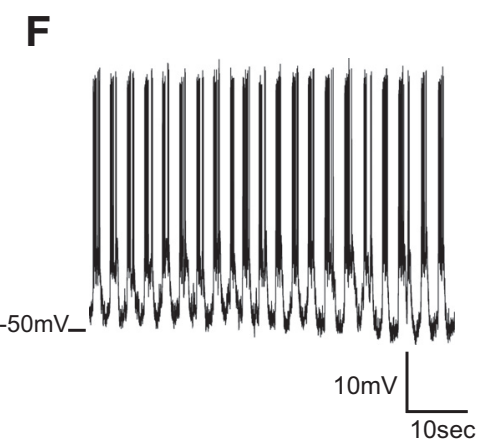

H

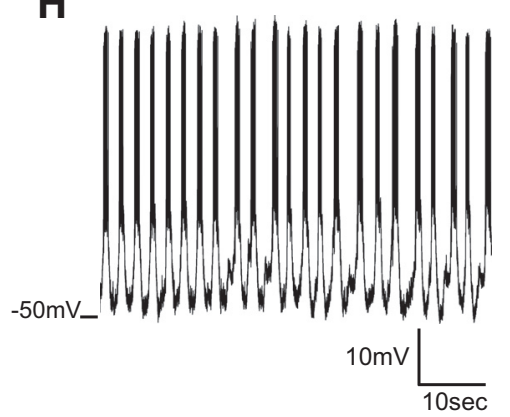

G

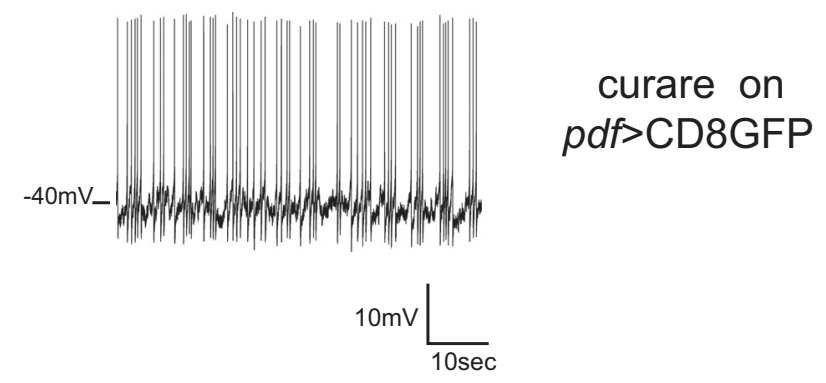

I

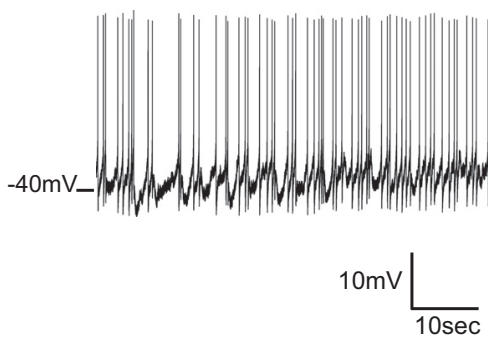

mecamylamine on $p d f>C D 8 G F P$

Figure 3. Blocking nicotinic cholinergic receptors triggers a firing switch. Bath application of the nicotinic antagonist curare (200 $\mu \mathrm{M})$ produced a change in the firing pattern of ILNvs from bursting to tonic. $A$, Initial minute of a quickly established recording of a control ( $p d f$-DsRed) ILNv firing APs in bursts with a frequency of 30 bursts/min. $B$, Upon bath application of curare (200 $\mu \mathrm{m}$ ), firing of the neuron becomes tonic, the membrane depolarizes, the AP firing rate decreases, and membrane oscillations disappear. $C$, Effect of curare is not reversible; however, in some examples, as the one included, membrane oscillations were partially restored and APs were reorganized in a pattern reminiscent of bursting activity. In this case, the bursting frequency decreased to 20 bursts/min, a reasonable decay taking into account that $10 \mathrm{~min}$ have passed since the beginning of the recording (related to Fig. 1O). D, Quantification of the membrane potential change produced by curare application comparing the trough of the membrane potential between bursts in the bursting mode, with the resting membrane potential of the tonic mode: $\mathrm{MP}_{\text {Basal }}=-45.7 \pm 1.7 \mathrm{mV}$,

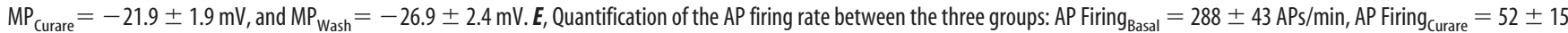
$\mathrm{APs} / \mathrm{min}$, and AP Firing Wash $_{1}=66 \pm 20 \mathrm{APs} / \mathrm{min}$. Different letters indicate significant differences $(p<0.05)$ after a one-way ANOVA with Tukey post hoc test. $n_{\text {Basal }}=6, n_{\text {Curare }}=6, n_{\text {Wash }}=5$. Genotype: pdf-DsRed. $\boldsymbol{F}, \mathbf{G}$, Firing switch is not genotype specific; $p d f>$ CD8GFP ILNvs become tonic upon bath perfusion of curare $200 \mu \mathrm{m}(n=8)$. $\boldsymbol{H}, \boldsymbol{I}$, Firing switch is recapitulated by perfusion of the noncompetitive nicotinic ACh receptor antagonist mecamylamine $(10 \mu \mathrm{m})$ on the $p d f>$ CD8GFP genotype $(n=3)$. $\boldsymbol{A}-\boldsymbol{C}, \boldsymbol{F}$ and $\boldsymbol{G}$, and $\boldsymbol{H}$ and $\boldsymbol{I}$ correspond to different parts of the same recording. Zeitgeber times of all recordings present in this figure were $2-10$ (daytime). 
A

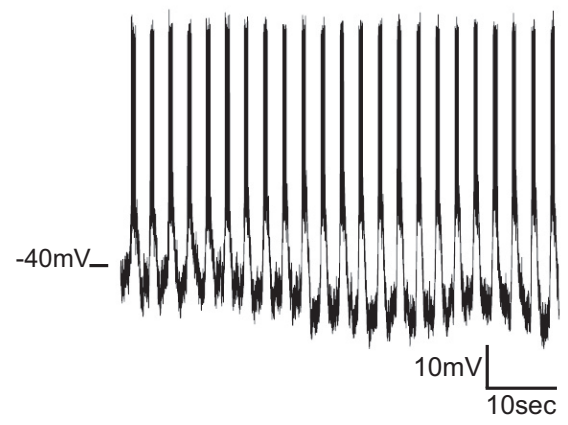

B

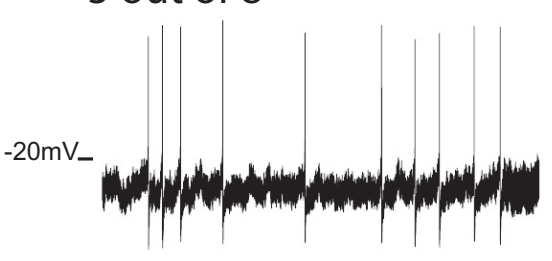

curare
C

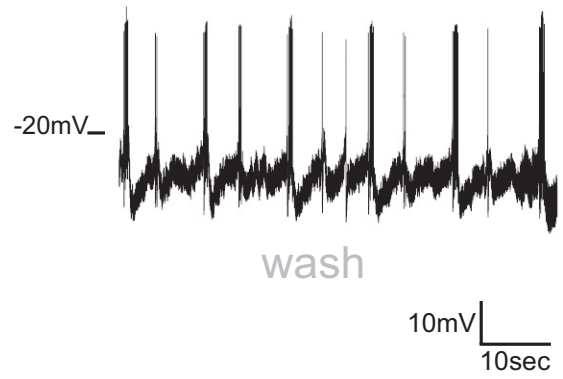

D

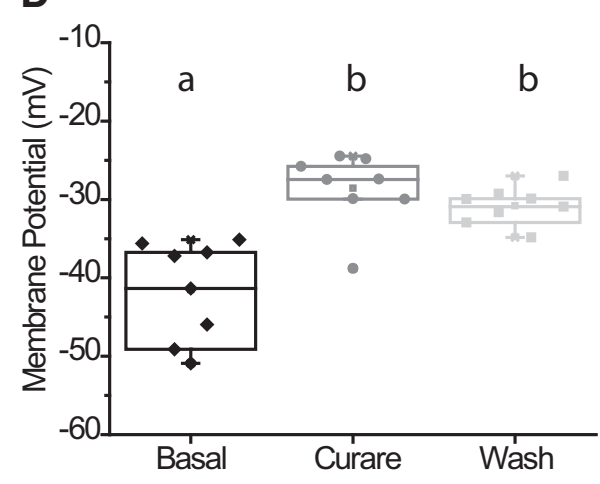

E

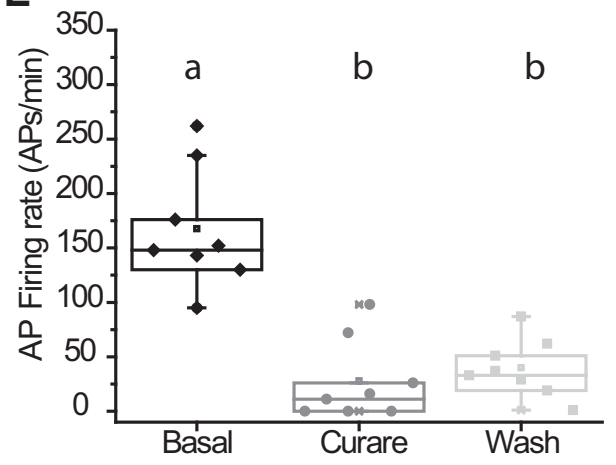

F

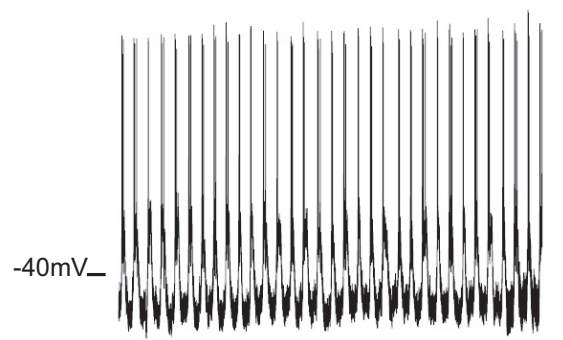

$10 \mathrm{mV}$
G

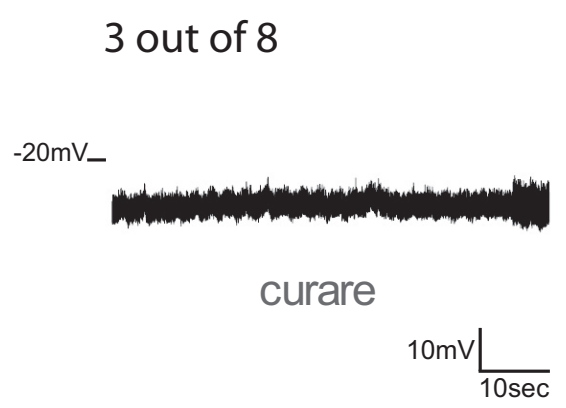

H

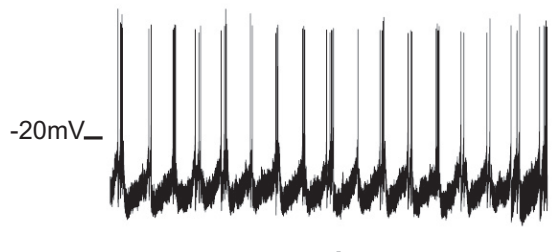

wash

$10 \mathrm{mV}$

$10 \mathrm{sec}$

Figure 4. Blocking nicotinic cholinergic receptors at nighttime switches from bursting to tonic firing, but can also silence ILNvs. Bath application of the nicotinic antagonist curare (200 $\mu \mathrm{M}$ ) on preparations of quickly dissected control ( $p d f$-DsRed) brains at nighttime produced a change in the firing pattern of ILNvs from bursting to tonic. $A$, Initial minute of a nighttime quickly established recording of a control ( $p d f$-DsRed) ILNv firing APs in bursts with a frequency of 23 bursts/min. B, Upon bath application of curare (200 $\mu \mathrm{M})$, firing becomes tonic, the membrane depolarizes, the AP firing rate decreases, and membrane oscillations disappear. C, Effect of curare is not reversible; however, some restoration of bursting activity is present in some preparations. $\boldsymbol{D}$, Quantification of the membrane potential change produced by curare application comparing the trough of the membrane potential between bursts in the bursting mode with the resting membrane potential of the tonic mode: $\mathrm{MP}_{\text {Basal }}=-41.5 \pm 2.4 \mathrm{mV}, \mathrm{MP}_{\text {Curare }}=-28.6 \pm 1.7 \mathrm{mV}$, and $\mathrm{MP}_{\text {Wash }}=-30.8 \pm 0.9 \mathrm{mV}$. E, Quantification of the AP firing rate between the three groups: AP Firing $g_{\text {Basal }}=168 \pm 21$ $\mathrm{APs} / \mathrm{min}, \mathrm{AP}$ Firing $\mathrm{Curare}=28 \pm 14 \mathrm{APs} / \mathrm{min}$, and AP Firing Wash $=40 \pm 10 \mathrm{APs} / \mathrm{min}$. Different letters indicate significant differences $(p<0.05)$ after a one-way ANOVA with Tukey post hoc test. $n_{\text {Basal }}=8, n_{\text {Curare }}=8, n_{\text {Wash }}=8$. F, Initial minute of a nighttime quickly established recording of a control ( pdf-DsRed) ILNv firing APs in bursts with a frequency of 32 bursts/min. $\mathbf{G}$, Upon bath application of curare $(200 \mu \mathrm{M})$, the membrane depolarizes and loses its oscillations and AP firing rate decreases to the point of being silent. $\boldsymbol{H}$, Effect of curare is not reversible; however, some restoration of bursting activity is regained in some preparations. Genotype: pdf-DsRed. $\boldsymbol{A}-\boldsymbol{C}$ and $\boldsymbol{F}$ - $\boldsymbol{H}$ correspond to different parts of the same recording. Zeitgeber times of all recordings present in this figure were $14-20$ (nighttime).

this, we used the UAS-GAL4 binary system (Brand and Perrimon, 1993 ) to express the heat-sensitive cation channel TrpA1 (Pulver et al., 2009) in L2 neurons and recorded electrophysiologically from lLNvs. The rationale behind this approach was to use a heat-inducible system to transiently overactivate L2 neurons, which would acutely result in the release of ACh. If L2 neurons were part of the circuit feeding information to the lLNvs, then we would expect a response in their physiology. To visualize lLNvs, the $p d f$-DsRed construct was included; therefore, the ge- notype of the experimental group was as follows: L2>TrpA1, $p d f$-DsRed (named TRP + HEAT). Nonpulsed genetic controls (named TRP + NO HEAT) and heat-pulsed controls lacking the TrpA1 channel (named CTROL + HEAT) were analyzed in parallel. The heat pulse was achieved by perfusing warmed external solution onto the brain preparation while recording.

We initially attempted to activate L2 neurons and record from ILNvs in quickly obtained preparations; however, we observed no change in their physiology under these conditions. We hypothe- 


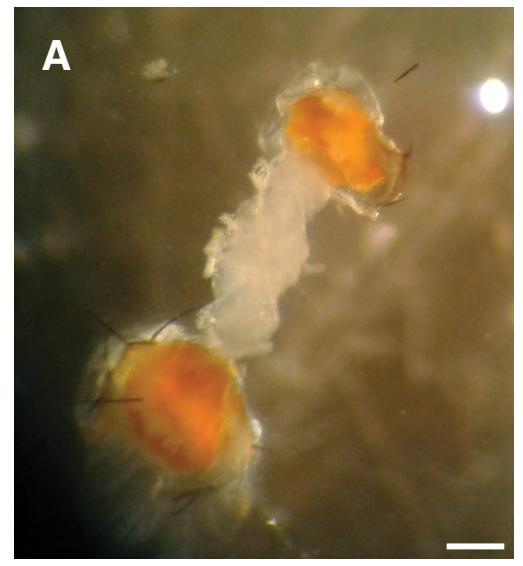

B

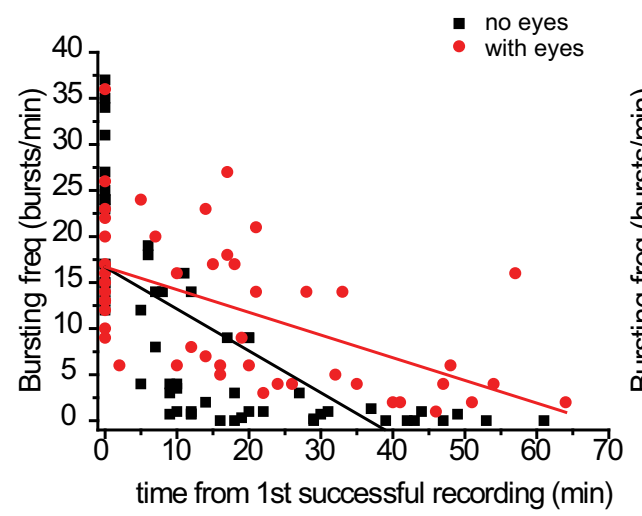

C

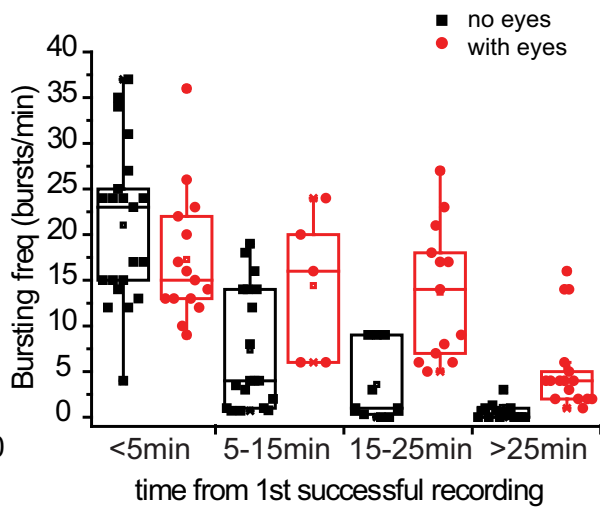

Figure 5. Synaptic input from the eye enables ILNv bursting behavior. A, Image of a novel brain preparation in which the eyes are preserved. Scale bar, $200 \mu \mathrm{m}$. $B$, Comparison of the frequency decay in the original and novel preparations. Two-way ANOVA statistical analysis indicates that, in both cases, bursting frequency decreases as a function of time $\left(F_{(1,72)}=18.07, p<0.01\right)$. However, the decay of bursting frequency in the novel preparation is significantly slowed down $\left(F_{(1,72)}=24.38, p<0.01\right): n_{\text {No eyes }}=67 ; n_{\text {with eyes }}=49$. Genotype: $p d f$-DsRed. C, Same data from $B$ plotted in a different way to enable a better appreciation of the properties of the bursting decay in the two preparations. No significant differences were found on the bursting frequency of early recordings ( $p=0.16)$. Zeitgeber time of recordings was distributed around the whole $24 \mathrm{~h}$ cycle.

sized that, in quickly recorded brains, $\mathrm{LNN}$ bursting frequency would be at its maximum and no further activation was possible. The fact that the initial bursting frequency was not different in with-eyes preparations from that in the no-eyes preparations (Fig. $5 C$ ) lent support to this hypothesis. To test it further, an alternative strategy was adopted; brain preparations were left in the perfusion chamber for 7-10 min to allow some decay in bursting frequency before activating L2 neurons. Under these conditions, all recorded lLNvs had a basal bursting frequency ranging from 7 to 27 bursts/min, which fits well with the variability of bursting decay observed for a larger population (Fig. 1C). Because of this variability, we normalized bursting frequency of each neuron to its basal. In a control situation in which there was no activation of L2 neurons (CTROL + HEAT), ILNvs showed, as expected, a progressive decay of bursting frequency (Fig. $6 \mathrm{~A}-$ C). However, upon activation of L2 neurons, lLNvs either increased or maintained their bursting frequency for a longer period of time. An example of an activated (TRP + HEAT) lLNv recording is shown in Figure 6, $D-F$, displaying a basal bursting frequency of 8 bursts/min (Fig. $6 D$ ) that increased to 14 bursts/ min upon perfusion of warm solution (Fig. 6E). The effect of activating L2 neurons persisted while the bathing solution was cooling down; in fact, in the example included, bursting had still not decayed below basal even after $8 \mathrm{~min}$ of recording. This observation fits nicely with the notion that $\mathrm{ACh}$ is a probursting signal for lLNvs. In addition, it is worth pointing out that this is the only condition that enabled us to record an increase in bursting frequency as opposed to a steady decay. Normalized and averaged change in bursting frequency of the three groups is shown in Figure $6 G$ (top), along with the profile of temperature change in the bath (bottom). We performed a two-way ANOVA analysis taking time and group (GENOTYPE + TREATMENT) as factors. The time factor was significant, indicating that bursting frequency decayed in the three groups $(p<0.01)$. However, this decay was not the same for all groups because the decay in bursting frequency of the activated group was significantly slower compared with either control group (for both, $p<0.01$ ). No significant differences were found between the two control groups ( $p=0.68$ ). These results suggest that $\mathrm{L} 2$ monopolar neurons are part of a circuit feeding visual information to lLNvs.

\section{L2 lamina neurons do not contact lLNvs directly}

To explore the possibility of a direct synaptic connection between L2 lamina neurons and lLNvs, we used the GRASP technique (Feinberg et al., 2008). Immunohistochemistry using an antibody specific for GFP reconstitution did not produce any signal in $p d f$-LexA $>$ LexAop-CD4spGFP ${ }^{11}, \mathrm{~L} 2>C D 4 s p G F P^{1-10}$ brains (data not shown), suggesting that the connection between L2 neurons and lLNvs is not direct and therefore intermediate synapses, including other neuron/s of the optic neuropiles, could mediate this communication.

In an attempt to find neuronal types that may interact with the ILNvs directly, we performed GRASP with two more lamina GAL4 drivers, one specific for the L1 neurons and the other for the Lai amacrine cell type. We also performed GRASP analysis with the commonly used photoreceptor driver Gmr-GAL4. We did not find GFP reconstitution between the lLNvs and the L1 ( $p d f$-LexA $>$ LexAop-CD4sp $G F P^{11}, \mathrm{~L} 1>C D 4 s p G F P^{1-10}$, data not shown) or the Lai neurons ( $p d f$-LexA $>$ LexAop-CD4spGFP ${ }^{11}$, Lai $>C D 4 s p G F P^{1-10}$, data not shown), suggesting that the interaction may lie further down in the visual processing circuit, probably in the medulla. Interestingly, we did find GFP reconstitution between the PDF-positive and the GMR domains ( $p d f$-LexA $>$ LexAop-CD4spGFP ${ }^{11}, G m r>C D 4 s p G F P^{1-10}$ ). This GRASP positive signal was localized onto the Hofbauer-Buchner (HB) eyelet tract (Fig. $6 \mathrm{H}$ ) and confirms that light information from this extraretinal organ reaches the LNvs, as has been suggested previously (Helfrich-Förster et al., 2002). Because the HB eyelet projects to the accessory medulla directly (Fig. 6I), without any synaptic relay on the lamina or medulla, this input pathway is independent of the one described here. Moreover, the location of the GFP reconstitution, in a region where the somas of both LNv types reside, cannot discriminate whether the HB eyelet synapses onto the lLNvs or the sLNvs. This positive GRASP result proves that, under our experimental conditions, it is possible to show reconstitution of GFP, thus validating the negative GRASP obtained with the L2, L1, and Lai drivers. All of these data together make a strong case for the L2-lLNv interaction being an indirect one. 

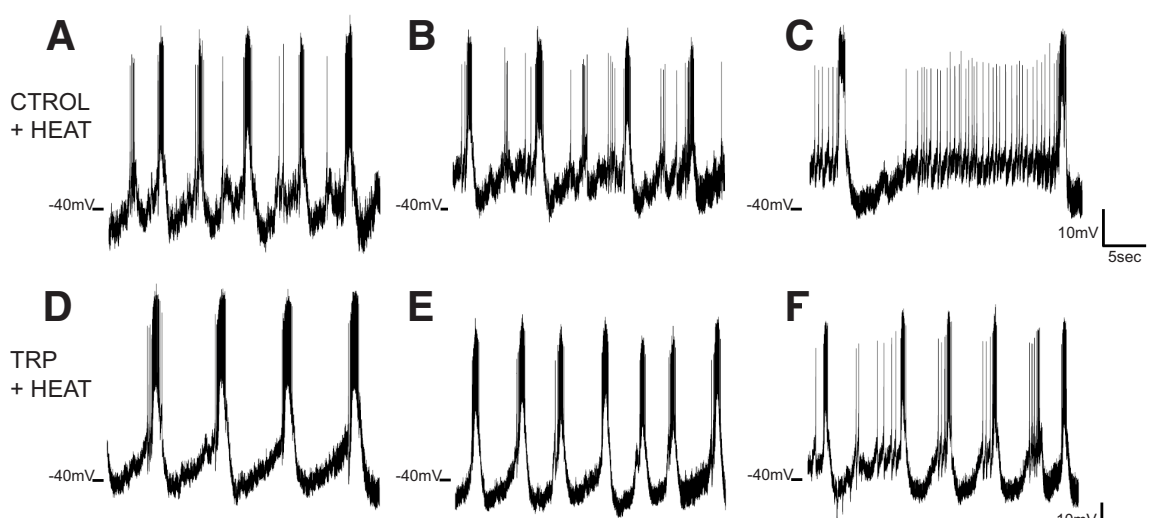

E Basal
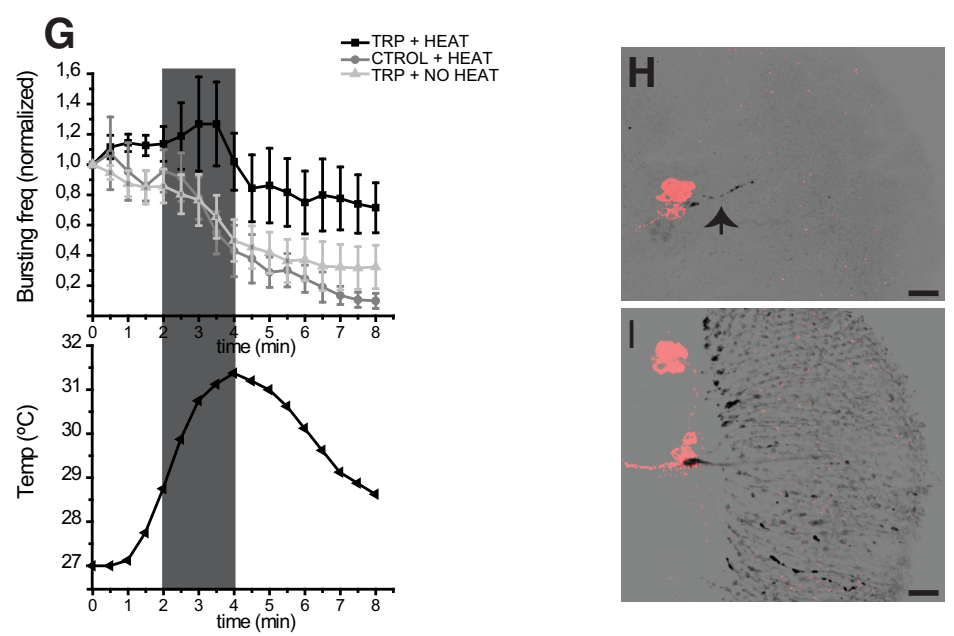

Figure 6. L2 neurons are part of the circuit that promotes ILNv bursting. To test the hypothesis of L2 lamina neurons being part of a neuronal circuit that feeds visual information to ILNvs, we acutely activated L2 neurons via genetic expression of the TrpA1 heat-activated channel and perfusion of warmed solution while simultaneously recording electrophysiologically from ILNvs. $\boldsymbol{A}-\boldsymbol{C}$, Representative recording of a ILNv of the control genotype ( $\mathrm{L} 2>+$, pdf-DsRed) that underwent a heat pulse. In this case, bursting frequency decays progressively from its basal 12 bursts/min to 10 bursts/min to 4 bursts/min in the 8 min spent perfusing the solutions. $\boldsymbol{D}-\boldsymbol{F}$, Representative recording of a ILNv of the experimental genotype (L2>TrpA1, pdf-DsRed) with a basal bursting frequency of 8 bursts/min, which increases to 14 bursts/min upon perfusion of warm solution. The effect of activating $L 2$ neurons persists while the bathing solution is cooling and, in this case, after $8 \mathrm{~min}$ of recording, bursting has not decreased below its basal yet. $\mathbf{G}$, Top, Bursting frequency decay profiles of the following groups are compared: TRP + HEAT refers to L2 $>$ TrpA1, pdf-DsRed with perfusion of warmed solution; TRP + NO HEAT refers to L2 > TrpA1, pdf-DsRed without perfusion of warmed solution; and CTROL + HEAT refers to $\mathrm{L} 2>+$, pdf-DsRed with perfusion of warmed solution. Bursting frequency was normalized to basal for each neuron and afterward averaged with the others in the same experimental group, Mean \pm SEM is shown. Two-way ANOVA statistical analysis taking the time as one factor and the group (GENOTYPE + TREATMENT) as the other indicates that bursting frequency decays in the three groups $\left(F_{(16,238)}=6.56, p<0.01\right)$. However, this decay is not the same for all groups $\left(F_{(2,238)}=\right.$ $35.23, p<0.01)$. Tukey post hoc analysis indicates that the decay in bursting frequency of the activated group is significantly slower than the decay in bursting frequency of either control $(p<0.01)$. No significant differences are found between the two control groups $(p=0.68)$. Bottom, Temperature profile in the chamber during the perfusion of warmed external solution. The time indicated in the bottom panel corresponds to those shown in the top panel. Gray area corresponds to the phase when bath

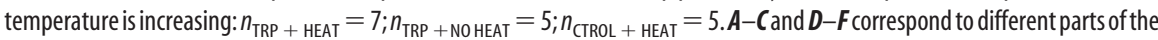
same recording. $\boldsymbol{H}$, GRASP analysis was performed to test for direct synaptic connections between different eye neuronal subtypes and LNvs. Among the neuronal types tested, only Gmr-GAL4 was able to reconstitute GFP in the context of $p d f$-LexA. The reconstituted signal was localized onto the HB tract (arrow). Genotype: $p d f$-LexA $>$ LexAop-CD4spGFP ${ }^{11}, G m r>C D 4 s p G F P^{1-10}$. Black indicates immunohistochemistry against reconstituted GFP; red, anti-PDF immunohistochemistry. Scale bar, $20 \mu \mathrm{m}$. I, Immunohistochemistry showing the two expression domains that reconstituted GFP in $\boldsymbol{H}$. Genotype: Gmr $>$ CD8GFP. Black indicates anti-GFP immunohistochemistry; red, anti-PDF immunohistochemistry. Scale bar, $20 \mu \mathrm{m}$.

L2 lamina neurons are part of a circuit that promotes arousal As we have shown in the present work, activation of L2 lamina neurons promotes $1 \mathrm{LNv}$ bursting activity (Fig. 6G). Moreover, high-frequency bursting has been associated with the release of neuropeptides (Dutton and Dyball, 1979; Liu et al., 2011). In addition, it has been reported that PDF neuropeptide functions as a sleepsuppressing signal when released from lLNvs (Chung et al., 2009). Taking all of this information together, we hypothesized that activation of $\mathrm{L} 2$ neurons would have a sleep-suppressing effect at the behavioral level.

To test this hypothesis, we quantified sleep before, during, and after activation of L2 neurons using the previously described TrpA1 heat-sensitive cation channel. Figure 7A shows the sleep profile of female flies of the experimental group as a function of the time of day (L2>Trp) together with its genetic controls $(+>\operatorname{Trp}$ and $\mathrm{L} 2>+)$. At $22^{\circ} \mathrm{C}$, the three genotypes showed the normal sleep pattern of Drosophila, in which sleep is present in the middle of the day (a nap), but is mainly concentrated at night (Fig. 7A, top). Upon increasing the temperature to $29^{\circ} \mathrm{C}$, the sleep pattern of all genotypes was altered (Fig. $7 A$, middle). Both control groups showed a sleep increase during the day (a natural response to a warmer day); conversely, the experimental group L2 $>$ Trp showed a significant sleep reduction, supporting our hypothesis. In L2 > Trp flies, sleep was strongly suppressed particularly during the first half of the day, suggesting that compensatory mechanisms may be recruited later on. However, compensation was not complete and sleep was less strongly but still significantly decreased during the night in L2>Trp flies compared with genetic controls. To better appreciate these effects, we calculated, for each individual fly, the difference in the amount of sleep (delta sleep) between induced $\left(29^{\circ} \mathrm{C}\right)$ and noninduced $\left(22^{\circ} \mathrm{C}\right)$ states. All sleep parameters assessed (total sleep, day sleep, and night sleep) were significantly reduced when L2 neurons were overactivated (Fig. $7 B-D$ ). The increase in temperature, although it affected sleep, did not alter the overall locomotor activity of the flies (Fig. 7E). After $24 \mathrm{~h}$ at $29^{\circ} \mathrm{C}$, the temperature was returned to $22^{\circ} \mathrm{C}$ to stop overactivation of $\mathrm{L} 2$ neurons (Fig. $7 A$, bottom). This had a striking effect on all sleep parameters of the experimental group, which showed a massive sleep rebound both during the day and at night. Figure 7 , $F-I$, shows the quantification of the 29$22^{\circ} \mathrm{C}$ delta sleep for all parameters examined. Figure 7 shows one representative experiment of three that retrieved similar results. The behavioral data shown here support the hypothesis of visual circuits providing excitatory synaptic input to lLNVs arousal neurons. 

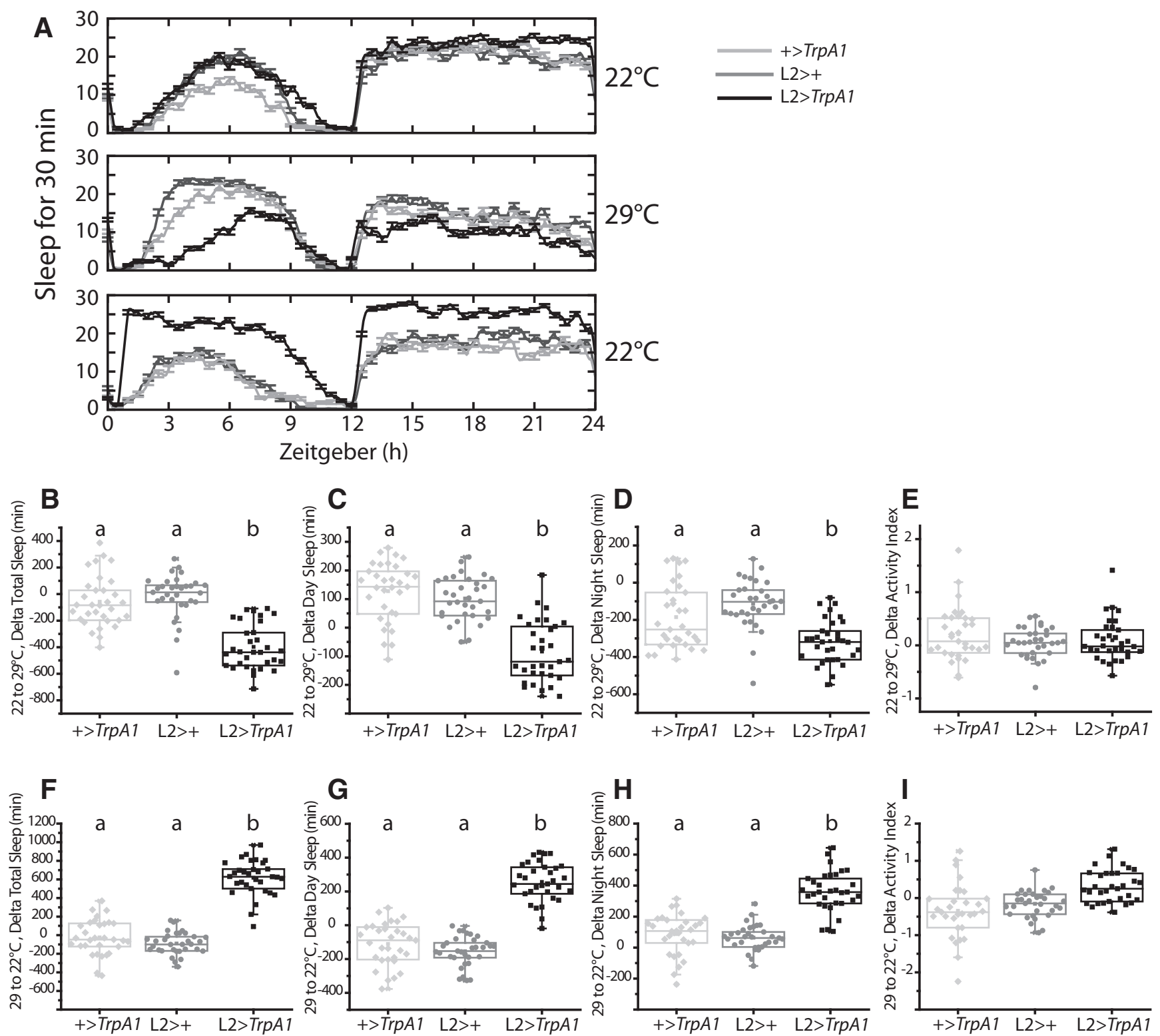

Figure 7. L2 lamina neurons are part of a circuit that promotes arousal. Sleep behavior was inferred from locomotor activity measured using Trikinetics DAM system before, during, and after overactivation of $\mathrm{L} 2$ neurons. $A$, Profiles of sleep for 30 min as a function of zeitgeber time for the experimental group $\mathrm{L} 2>\operatorname{Trp}$ and genetic controls; $+>\operatorname{Trp}$ and $\mathrm{L} 2>+$. Top, At $22^{\circ} \mathrm{C}$, all genotypes show a similar and normal sleep profile. Middle, $A t 29^{\circ} \mathrm{C}$, sleep is affected but in different ways. In both controls, the increase in temperature is accompanied by an increase in day sleep. On the contrary, in the experimental group, the increased temperature produces overexcitation of $L 2$ neurons and this generates a marked reduction of sleep during the day and a less abrupt but still significant reduction of sleep at night. Bottom, at $22^{\circ} \mathrm{C}$, the experimental group shows an important sleep rebound. $\boldsymbol{B}-\boldsymbol{E}$, Sleep and activity parameters were calculated for each individual and the delta between the $29^{\circ} \mathrm{C}$ day and the initial $22^{\circ} \mathrm{C}$ day were plotted in box charts for the following: total sleep $(\boldsymbol{B})$, day sleep $(\boldsymbol{C})$, night sleep $(\boldsymbol{D})$, and activity index $(\boldsymbol{E})$. $\boldsymbol{F}-\boldsymbol{I}$, Sleep and activity parameters were calculated for each individual and the delta between the recovery day at $22^{\circ} \mathrm{C}$ and the activation day at $29^{\circ} \mathrm{C}$ were plotted in box charts for the following: total sleep $(\boldsymbol{F})$, day sleep $(\boldsymbol{G})$, night sleep $(\boldsymbol{H})$, and activity index $(\boldsymbol{I})$. Different letters indicate significant differences $(p<0.05)$ after a one-way ANOVA with Tukey post hoc test. $n_{+>\operatorname{Trp}}=31, n_{\mathrm{L} 2>+}=32, n_{\mathrm{L} 2>\operatorname{Trp}}=32$. Shown is one representative experiment of $N=3$.

\section{Discussion}

To fire or not to fire, that is the question...

Here, we reveal that lLNv activity depends on inputs from cholinergic visual processing centers. To our surprise, all lLNvs were found spontaneously firing APs even when kept ex vivo for $>1 \mathrm{~h}$. In contrast, Cao et al. (2008) reported that $\sim 35 \%$ of the lLNvs recorded were silent, which is reminiscent of the proportion of silent neurons found when recording from the mammalian $\mathrm{SCN}$, somehow validating their findings. However, in those studies, the recorded neurons belonged to heterogeneous populations of SCN neurons (Walsh et al., 1992; Kim and Dudek, 1993), clearly not comparable to a more homogeneous cluster as is the case of the lLNvs. Our data show that, under certain recording conditions, all lLNvs can be found in a spontaneously active state and, furthermore, if recordings are established quickly, then they all fire APs in a bursting mode.

The finding that bursting decays as a function of time is indeed intriguing and might account for some of the inconsistencies previously ascribed to the inherent complexity of the approach (which is accompanied by a rather low number of observations under each condition). In fact, the variability of the bursting frequency decay at first led us to believe that the bursting and the tonic were two different neuronal populations, when in fact the tonic neurons were the result of bursting decay. In addition, 
we also found daily variation in the ratio of bursting to tonic neurons when the time from dissection to recording was not taken into account, reinforcing the notion that there is circadian variation in firing pattern. However, as more experiments were performed and the time from dissection to recordings was reduced effectively, tonic neurons became more difficult to find, even among those recorded at nighttime. In sum, although we endorse the idea of daily variation of firing frequency of lLNvs, our results are consistent with information encoded as a daily variation of bursting frequency rather than in a drastic change of firing mode. Ultimately, the end result would be similar: during the day, a higher frequency of ILNv bursting would result in increased release of PDF and, at night, lower bursting frequencies would result in decreased release of the arousal signal.

\section{lLNvs, an integration center controlling arousal}

Light is evidently an arousing cue for diurnal animals, so it is not surprising that light produces an increase in activity of lLNvs, cells that are now recognized to play a role as arousal neurons. Light excites LNvs by at least two mechanisms, cell autonomously via the expression of the photoreceptor protein CRYPTOCHROME (CRY) (Fogle et al., 2011) and indirectly via the extraretinal and retinal photoreceptors, as postulated numerous times in the past based on anatomical (Helfrich-Förster et al., 2002; Malpel et al., 2002; Helfrich-Förster et al., 2007) and behavioral (Cusumano et al., 2009) data and as demonstrated in the current work. However, light is not the only important signal capable of modulating ILNv activity. ILNvs have been proposed as a control center integrating both external (environmental) and internal (from the physiological state) information and therefore are known to get information from many sources, including: (1) the circadian time, defined by its own molecular clock but also synaptically through peptidergic neurotransmission from the sLNvs (Shang et al., 2013); (2) a still to be defined GABAergic neuronal circuit governing sleep homeostasis (Agosto et al., 2008; Parisky et al., 2008; Gmeiner et al., 2013); and (3) social interaction through as yet unknown neuronal circuits (Donlea et al., 2009; Donlea et al., 2014).

Consistent with their proposed role as an integration center, it is not surprising that lLNvs express receptors for a wide array of neurotransmitters and neuropeptides, including GABA (Parisky et al., 2008; McCarthy et al., 2011; Li et al., 2013), ACh (McCarthy et al., 2011), glutamate (McCarthy et al., 2011), dopamine (Shang et al., 2011), octopamine (Shang et al., 2011), histamine (Hong et al., 2006), and short neuropeptide F (Shang et al., 2013). In contrast to their many described synaptic inputs, so far, the only well defined neuronal output of lLNvs is the neuropeptide PDF. This input to output imbalance reinforces the idea of lLNvs playing a role as an integrator of many cues. The cellular targets of the PDF released by the lLNvs are still unknown, probably because of the widespread localization of PDFR (Im and Taghert, 2010) and the volumetric transmission of peptide neurotransmitters (Nässel, 2009). Finding this would be a crucial step in the unraveling of the sleep-arousal circuit of Drosophila.

\section{Visual circuit presynaptic to lLNvs}

As mentioned, light is detected cell autonomously by ILNVs through CRY (Fogle et al., 2011), so what is the purpose of an additional method for light detection? We can envision at least two possible scenarios. The obvious one is that light sensing is of paramount importance for arousal neurons such as ILNvs, so they may count with redundant mechanisms to ensure this. Another possibility is that the information that lLNvs get from a visual circuit that involves L2 lamina neurons is not light per se, but already processed visual information. In other words, through this pathway, ILNvs may not only see light, but also sense motion from visual stimuli. Such visual information and the existence of visual circuits presynaptic to lLNVs have been proposed to affect their synaptic plasticity in the context of social enrichment (Donlea and Shaw, 2009).

The development of an eyes-attached brain preparation has allowed us to recognize them as the source of the probursting signal because lLNv bursting decay in this novel preparation is significantly delayed compared with the original one. It is worth pointing out that two different ILNv populations might be perceived in the with-eyes preparation regarding the kinetics of their bursting decay. Although all lLNvs display a slower bursting decay compared with the no-eyes preparation, some lLNvs in the eyes-attached one show a faster bursting decay than others. Two potential explanations could account for this result. The first possibility is that the inherent technical difficulty associated with the dissection leading to cells with slower bursting decay corresponds to preparations in which connectivity between the eyes and optic neuropiles was best preserved. Alternatively, ILNvs might be not homogenously connected to the visual system and only some of them might receive more direct probursting inputs from visual centers. Additional experiments would be required to discern between these possibilities.

Our results describe a connection between the L 2 feedforward lamina monopolar cells and lLNvs. However, many lines of evidence point to an indirect connection between these two cell types. First, we saw no GRASP reconstitution between them. Moreover, although, in specific conditions, unidirectional motion detection is determined at the level of lamina neurons such as L2 (Rister et al., 2007), higher computation of motion depends on lamina neurons passing on information to the second optic neuropile, the medulla (Tuthill et al., 2013). Our results are more consistent with $\mathrm{lLNv}$ receiving information from interneurons in the medulla because ILNvs projections do not reach the lamina, as is the case for other clock neurons (Damulewicz and Pyza, 2011). Furthermore, because we bath applied the cholinergic antagonists, the effect of these pharmacological treatments could have affected cholinergic neurons in a multisynaptic visual circuit feeding information onto lLNvs. ACh is the predominant excitatory neurotransmitter in the optic neuropiles and $>40$ different cholinergic neuronal types have been identified (Varija Raghu et al., 2011). Given the complexity of visual circuits and the prevalence of ACh as their neurotransmitter of choice, it is difficult to predict where the ACh that promotes bursting of lLNvs is coming from and what it is acting upon. At this point, we propose that there is a neuronal circuit connecting L2 neurons with ILNvs and that ACh is involved in at least one step that is crucial for the overall effect of this circuit: to promote bursting of lLNvs. Further research will be needed to unravel the presynaptic visual input to the lLNvs. The recent development of genetic tools to circumscribe gene expression to even more restricted subgroups will be useful in this endeavor (Tuthill et al., 2013).

\section{Concluding remarks}

Here, we reveal the properties of a Drosophila brain preparation that has been used in the past for electrophysiological recording of lLNvs. We have established that ACh is a key neurotransmitter that participates in a circuit upstream of lLNvs, which includes L2 monopolar neurons of the lamina, promotes bursting of ILNvs, and suppresses sleep. Moreover, we describe herein a new brain 
preparation in which the eyes are preserved and demonstrate that this has an impact on lLNv physiology.

\section{References}

Agosto J, Choi JC, Parisky KM, Stilwell G, Rosbash M, Griffith LC (2008) Modulation of GABAA receptor desensitization uncouples sleep onset and maintenance in Drosophila. Nat Neurosci 11:354-359. CrossRef Medline

Brand AH, Perrimon N (1993) Targeted gene expression as a means of altering cell fates and generating dominant phenotypes. Development 118: 401-415. Medline

Cao G, Nitabach MN (2008) Circadian control of membrane excitability in Drosophila melanogaster lateral ventral clock neurons. J Neurosci 28: 6493-6501. CrossRef Medline

Cao G, Platisa J, Pieribone VA, Raccuglia D, Kunst M, Nitabach MN (2013) Genetically targeted optical electrophysiology in intact neural circuits. Cell 154:904-913. CrossRef Medline

Chung BY, Kilman VL, Keath JR, Pitman JL, Allada R (2009) The GABA(A) receptor RDL acts in peptidergic PDF neurons to promote sleep in Drosophila. Curr Biol 19:386-390. Medline

Colwell CS (2011) Linking neural activity and molecular oscillations in the SCN. Nat Rev Neurosci 12:553-569. CrossRef Medline

Cusumano P, Klarsfeld A, Chélot E, Picot M, Richier B, Rouyer F (2009) PDF-modulated visual inputs and cryptochrome define diurnal behavior in Drosophila. Nat Neurosci 12:1431-1437. CrossRef Medline

Damulewicz M, Pyza E (2011) The clock input to the first optic neuropil of Drosophila melanogaster expressing neuronal circadian plasticity. PLoS One 6:e21258. CrossRef Medline

Depetris-Chauvin A, Berni J, Aranovich EJ, Muraro NI, Beckwith EJ, Ceriani MF (2011) Adult-specific electrical silencing of pacemaker neurons uncouples molecular clock from circadian outputs. Curr Biol 21:1783-1793. CrossRef Medline

Donlea JM, Shaw PJ (2009) Sleeping together using social interactions to understand the role of sleep in plasticity. Adv Genet 68:57-81. CrossRef Medline

Donlea JM, Ramanan N, Shaw PJ (2009) Use-dependent plasticity in clock neurons regulates sleep need in Drosophila. Science 324:105-108. CrossRef Medline

Donlea JM, Ramanan N, Silverman N, Shaw PJ (2014) Genetic rescue of functional senescence in synaptic and behavioral plasticity. Sleep 37: 1427-1437. Medline

Dutton A, Dyball RE (1979) Phasic firing enhances vasopressin release from the rat neurohypophysis. J Physiol 290:433-440. CrossRef Medline

Feinberg EH, Vanhoven MK, Bendesky A, Wang G, Fetter RD, Shen K, Bargmann CI (2008) GFP Reconstitution Across Synaptic Partners (GRASP) defines cell contacts and synapses in living nervous systems. Neuron 57: 353-363. CrossRef Medline

Fogle KJ, Parson KG, Dahm NA, Holmes TC (2011) CRYPTOCHROME is a blue-light sensor that regulates neuronal firing rate. Science 331: 1409-1413. CrossRef Medline

Gilestro GF, Cirelli C (2009) pySolo: a complete suite for sleep analysis in Drosophila. Bioinformatics 25:1466-1467. CrossRef Medline

Gmeiner F, Kołodziejczyk A, Yoshii T, Rieger D, Nässel DR, Helfrich-Förster C (2013) GABA(B) receptors play an essential role in maintaining sleep during the second half of the night in Drosophila melanogaster. J Exp Biol 216:3837-3843. CrossRef Medline

Gorostiza EA, Depetris-Chauvin A, Frenkel L, Pírez N, Ceriani MF (2014) Circadian pacemaker neurons change synaptic contacts across the day. Curr Biol 24:2161-2167. CrossRef Medline

Hardin PE (2011) Molecular genetic analysis of circadian timekeeping in Drosophila. Adv Genet 74:141-173. CrossRef Medline

Helfrich-Förster C, Edwards T, Yasuyama K, Wisotzki B, Schneuwly S, Stanewsky R, Meinertzhagen IA, Hofbauer A (2002) The extraretinal eyelet of Drosophila: development, ultrastructure, and putative circadian function. J Neurosci 22:9255-9266. Medline

Helfrich-Förster C, Shafer OT, Wülbeck C, Grieshaber E, Rieger D, Taghert P (2007) Development and morphology of the clock-gene-expressing lateral neurons of Drosophila melanogaster. J Comp Neurol 500:47-70. CrossRef Medline

Hendricks JC, Finn SM, Panckeri KA, Chavkin J, Williams JA, Sehgal A, Pack AI (2000) Rest in Drosophila is a sleep-like state. Neuron 25:129-138. CrossRef Medline
Hong ST, Bang S, Paik D, Kang J, Hwang S, Jeon K, Chun B, Hyun S, Lee Y, Kim J (2006) Histamine and its receptors modulate temperaturepreference behaviors in Drosophila. J Neurosci 26:7245-7256. CrossRef Medline

Im SH, Taghert PH (2010) PDF receptor expression reveals direct interactions between circadian oscillators in Drosophila. J Comp Neurol 518: 1925-1945. CrossRef Medline

Kim YI, Dudek FE (1993) Membrane properties of rat suprachiasmatic nucleus neurons receiving optic nerve input. J Physiol 464:229-243. CrossRef Medline

Kolodziejczyk A, Sun X, Meinertzhagen IA, Nässel DR (2008) Glutamate, GABA and acetylcholine signaling components in the lamina of the Drosophila visual system. PLoS One 3:e2110. CrossRef Medline

Lelito KR, Shafer OT (2012) Reciprocal cholinergic and GABAergic modulation of the small ventrolateral pacemaker neurons of Drosophila's circadian clock neuron network. J Neurophysiol 107:2096-2108. CrossRef Medline

Li Y, Zhou Z, Zhang X, Tong H, Li P, Zhang ZC, Jia Z, Xie W, Han J (2013) Drosophila neuroligin 4 regulates sleep through modulating GABA transmission. J Neurosci 33:15545-15554. CrossRef Medline

Lin Y, Stormo GD, Taghert PH (2004) The neuropeptide pigmentdispersing factor coordinates pacemaker interactions in the Drosophila circadian system. J Neurosci 24:7951-7957. CrossRef Medline

Liu S, Lamaze A, Liu Q, Tabuchi M, Yang Y, Fowler M, Bharadwaj R, Zhang J, Bedont J, Blackshaw S, Lloyd TE, Montell C, Sehgal A, Koh K, Wu MN (2014) WIDE AWAKE mediates the circadian timing of sleep onset. Neuron 82:151-166. CrossRef Medline

Liu X, Porteous R, d'Anglemont de Tassigny X, Colledge WH, Millar R, Petersen SL, Herbison AE (2011) Frequency-dependent recruitment of fast amino acid and slow neuropeptide neurotransmitter release controls gonadotropin-releasing hormone neuron excitability. J Neurosci 31: 2421-2430. CrossRef Medline

Malpel S, Klarsfeld A, Rouyer F (2002) Larval optic nerve and adult extraretinal photoreceptors sequentially associate with clock neurons during Drosophila brain development. Development 129:1443-1453. Medline

McCarthy EV, Wu Y, Decarvalho T, Brandt C, Cao G, Nitabach MN (2011) Synchronized bilateral synaptic inputs to Drosophila melanogaster neuropeptidergic rest/arousal neurons. J Neurosci 31:8181-8193. CrossRef Medline

Muraro NI, Pírez N, Ceriani MF (2013) The circadian system: plasticity at many levels. Neuroscience 247:280-293. CrossRef Medline

Nässel DR (2009) Neuropeptide signaling near and far: how localized and timed is the action of neuropeptides in brain circuits? Invert Neurosci 9:57-75. CrossRef Medline

Ozkaya O, Rosato E (2012) The circadian clock of the fly: a neurogenetics journey through time. Adv Genet 77:79-123. CrossRef Medline

Parisky KM, Agosto J, Pulver SR, Shang Y, Kuklin E, Hodge JJ, Kang K, Kang K, Liu X, Garrity PA, Rosbash M, Griffith LC (2008) PDF cells are a GABA-responsive wake-promoting component of the Drosophila sleep circuit. Neuron 60:672-682. CrossRef Medline

Park D, Griffith LC (2006) Electrophysiological and anatomical characterization of PDF-positive clock neurons in the intact adult Drosophila brain. J Neurophysiol 95:3955-3960. CrossRef Medline

Park JH, Helfrich-Förster C, Lee G, Liu L, Rosbash M, Hall JC (2000) Differential regulation of circadian pacemaker output by separate clock genes in Drosophila. Proc Natl Acad Sci U S A 97:3608-3613. CrossRef Medline

Pollack I, Hofbauer A (1991) Histamine-like immunoreactivity in the visual system and brain of Drosophila melanogaster. Cell Tissue Res 266: 391-398. CrossRef Medline

Pulver SR, Pashkovski SL, Hornstein NJ, Garrity PA, Griffith LC (2009) Temporal dynamics of neuronal activation by Channelrhodopsin-2 and TRPA1 determine behavioral output in Drosophila larvae. J Neurophysiol 101:3075-3088. CrossRef Medline

Rister J, Pauls D, Schnell B, Ting CY, Lee CH, Sinakevitch I, Morante J, Strausfeld NJ, Ito K, Heisenberg M (2007) Dissection of the peripheral motion channel in the visual system of Drosophila melanogaster. Neuron 56:155-170. CrossRef Medline

Schindelin J, Arganda-Carreras I, Frise E, Kaynig V, Longair M, Pietzsch T, Preibisch S, Rueden C, Saalfeld S, Schmid B, Tinevez JY, White DJ, Hartenstein V, Eliceiri K, Tomancak P, Cardona A (2012) Fiji: an opensource platform for biological-image analysis. Nat Methods 9:676-682. CrossRef Medline

Seluzicki A, Flourakis M, Kula-Eversole E, Zhang L, Kilman V, Allada R 
(2014) Dual PDF signaling pathways reset clocks via TIMELESS and acutely excite target neurons to control circadian behavior. PLoS Biol 12:e1001810. CrossRef Medline

Shang Y, Griffith LC, Rosbash M (2008) Light-arousal and circadian photoreception circuits intersect at the large PDF cells of the Drosophila brain. Proc Natl Acad Sci U S A 105:19587-19594. CrossRef Medline

Shang Y, Haynes P, Pírez N, Harrington KI, Guo F, Pollack J, Hong P, Griffith LC, Rosbash M (2011) Imaging analysis of clock neurons reveals light buffers the wake-promoting effect of dopamine. Nat Neurosci 14: 889-895. CrossRef Medline

Shang Y, Donelson NC, Vecsey CG, Guo F, Rosbash M, Griffith LC (2013) Short neuropeptide F is a sleep-promoting inhibitory modulator. Neuron 80:171-183. CrossRef Medline

Shaw PJ, Cirelli C, Greenspan RJ, Tononi G (2000) Correlates of sleep and waking in Drosophila melanogaster. Science 287:1834-1837. CrossRef Medline

Sheeba V, Gu H, Sharma VK, O’Dowd DK, Holmes TC (2008a) Circadianand light-dependent regulation of resting membrane potential and spontaneous action potential firing of Drosophila circadian pacemaker neurons. J Neurophysiol 99:976-988. CrossRef Medline

Sheeba V, Fogle KJ, Kaneko M, Rashid S, Chou YT, Sharma VK, Holmes TC (2008b) Large ventral lateral neurons modulate arousal and sleep in Drosophila. Curr Biol 18:1537-1545. CrossRef Medline
Tabuchi M, Lone SR, Liu S, Liu Q, Zhang J, Spira AP, Wu MN (2015) Sleep interacts with abeta to modulate intrinsic neuronal excitability. Curr Biol 25:702-712. CrossRef Medline

Takemura SY, Lu Z, Meinertzhagen IA (2008) Synaptic circuits of the Drosophila optic lobe: the input terminals to the medulla. J Comp Neurol 509:493-513. CrossRef Medline

Tuthill JC, Nern A, Holtz SL, Rubin GM, Reiser MB (2013) Contributions of the 12 neuron classes in the fly lamina to motion vision. Neuron 79: 128-140. CrossRef Medline

Varija Raghu S, Reiff DF, Borst A (2011) Neurons with cholinergic phenotype in the visual system of Drosophila. J Comp Neurol 519:162-176. CrossRef Medline

Walsh IB, van den Berg RJ, Marani E, Rietveld WJ (1992) Spontaneous and stimulated firing in cultured rat suprachiasmatic neurons. Brain Res 588: 120-131. CrossRef Medline

Wegener C, Hamasaka Y, Nässel DR (2004) Acetylcholine increases intracellular $\mathrm{Ca} 2+$ via nicotinic receptors in cultured PDF-containing clock neurons of Drosophila. J Neurophysiol 91:912-923. Medline

Welsh DK, Takahashi JS, Kay SA (2010) Suprachiasmatic nucleus: cell autonomy and network properties. Annu Rev Physiol 72:551-577. CrossRef Medline 